\title{
Mortalidad entre Jóvenes Inmigrantes Nicaragüenses en Costa Rica, 2000-2017
}

\author{
Mortality of young Nicaraguan inmigrants in Costa Rica during the 2000-2017 period
}

Roger E. Bonilla-Carrión ${ }^{1,2 *}$ orcid.org/0000-0002-8789-4494

\author{
1. Centro Centroamericano de Población, Universidad de Costa Rica. San José, Costa Rica \\ 2. Escuela de Estadística, Universidad de Costa Rica. San José, Costa Rica
}

\section{Resumen}

Introducción: La pobreza, discriminación y el aislamiento social, son elementos que afectan a las personas inmigrantes nicaragüenses residentes en Costa Rica, condiciones que pueden afectar las tasas de mortalidad observada en esta población migrante. Objetivo: Determinar las tasas de mortalidad por enfermedades y causas externas en población joven inmigrante nicaragüense y nativos costarricenses en Costa Rica. Materiales y métodos: Con la información de defunciones entre el año 2000 y 2017 y la población del Censo 2011 se calcularon tasas de mortalidad y posteriormente el riesgo relativo (RR) de diferentes causas de mortalidad. Los resultados se compararon con un estudio que analizó este fenómeno entre 1998-2008. Resultados: El $64 \%$ de las defunciones de inmigrantes son por causas externas, versus el $57 \%$ de los locales. Con respecto a los datos de hace 10 años, hay una disminución de 2 puntos porcentuales de las defunciones por causas externas entre los inmigrantes. Los inmigrantes tienen RR mayores de mortalidad por causas externas (otros accidentes 1,85, homicidios 1,72, accidentes vehiculares 1,22 y suicidios 1,14). Se destaca una disminución de 0,27 y 0,15 del RR de los homicidios y alcoholismo. Conclusiones: Los patrones de mortalidad de los inmigrantes empiezan comportarse como el de la población costarricense.

Palabras clave: Inmigrantes; mortalidad; causas externas; riesgo relativo. (Fuente: DeCS, Bireme).

\begin{abstract}
Introduction: Poverty, discrimination, and social isolation are some elements that affect Nicaraguan immigrants residing in Costa Rica, conditions that have an impact on the mortality figures observed in this migrant population. Objective: To determine mortality rates caused by diseases and external reasons in both the young Nicaraguan immigrant population and native Costa Rican natives. Materials and methods: Data on deaths between 2000 and 2017 and the 2011 Census information of the Costa Rican population were used to calculate mortality rates and the relative risk (RR) associated with different causes of mortality. Results were compared to a study that analyzed this phenomenon between 1998-2008. Results: Compared to the deaths in the Costa Rican population (57\%), 64\% of deaths in immigrants are due to external causes. With respect to the data from 10 years ago, there is a 2-percentage point reduction in deaths of immigrants caused by external reasons. Immigrants show higher RRs of mortality due to external causes (other accidents 1.85 , homicides 1.72, car accidents 1.22, and suicide 1.14). A reduction of 2.27 and 0.15 in the RR of homicides and alcoholism, respectively, is highlighted. Conclusions: Mortality patterns in immigrants begin to behave as those of the Costa Rican population.
\end{abstract}

Keywords: Inmigrants; mortality; injury; relative risk. (Fuente: DeCS, Bireme).

\footnotetext{
*Autor de correspondencia

Roger E. Bonilla

e-mail: roger.bonilla@ucr.ac.cr
} 


\section{Introducción}

En décadas recientes, la migración de nicaragüenses a Costa Rica fue la más alta en la historia del país ${ }^{(1,2) .}$ El X Censo de Población y Vivienda 2011 muestra que más de la mitad de los inmigrantes nicaragüenses son jóvenes, entre 15 y 34 años ${ }^{(3,4)}$. La reciente migración de jóvenes nicaragüenses hacia Costa Rica se debe principalmente a razones económicas, la población joven busca incorporarse al mercado laboral en sectores en donde se requiere una cantidad importante de mano de obra, particularmente la agricultura(6).

Debido a la selectividad del fenómeno migratorio, los inmigrantes nicaragüenses tienen características similares a otras minorías de inmigrantes en otros países: baja mortalidad y morbilidad en muertes por enfermedades y alta mortalidad en causas externas (particularmente en accidentes y homicidios) como lo presentado por Bonilla y Chavarria(1) $\mathrm{y}$ Herring et al.,(5) para el caso de Costa Rica; Shor et al.,(7) y Aldridge et al.,(8) para el caso estadounidense; Ikram et al.,(9) para el caso europeo.

El caso latinoamericano tiene características particulares, aunque hay grandes intercambios migratorios en la región, los diferenciales de mortalidad entre los inmigrantes y la población local en el contexto de la migración "Sur-Sur" generalmente sigue con poco estudio(5). Para Chile, se ha identificado un exceso de casos de tuberculosis entre los inmigrantes, sin que eso llegue a constituir un riesgo especial para la población general(10).

En Costa Rica, hasta la fecha los estudios de Herring et al.,(5) y el de Herring y Bonilla(11) han investigado la mortalidad general de los inmigrantes nicaragüenses. Ambos estudios analizaron las muertes violentas en ese país y encontraron que el grupo de edad de 15-34 años es el más afectado(5). Bonilla y Chavarría(1) realizaron el estudio más exhaustivo de la mortalidad por causas externas y por enfermedades entre inmigrantes nicaragüenses jóvenes en Costa Rica y la compararon con la población costarricense. Los autores concluyeron que los inmigrantes tienen riesgos relativos (RR) mayores de mortalidad por causas externas que su correspondiente costarricense (homicidios $R R=2,00$, otros accidentes $R R=1,70$ y accidentes vehiculares $\mathrm{RR}=1,17)$. En particular, se encontró que los inmigrantes nicaragüenses tienen el doble de riesgo de morir a causa de homicidios que su contraparte costarricense.

Acuña et al.,(12) en 2003 concluyeron que el perfil de la mortalidad de la población inmigrante presenta patrones similares al de la población costarricense que evidencia un aumento de muertes por enfermedades crónicas y traumatismos. Sin embargo, las muertes por causas violentas $y$ accidentes en la población nicaragüense merecen atención especial.

A pesar de la importancia de la población inmigrante en la economía y en la sociedad costarricense, es muy poca la información que se ha logrado producir para definir las características de salud de esta población(12). Por ello el objetivo de este estudio fue determinar las tasas de mortalidad por enfermedades y causas externas en población joven inmigrante nicaragüense y nativos costarricenses en Costa Rica. Por la naturaleza descriptiva del estudio, esta es una comparación demográfica y no procura explicar los factores sociales para la migración y las condiciones de salubridad de los inmigrantes y políticas sociales que pudieran explicar los resultados.

El estudio de la mortalidad entre jóvenes inmigrantes en Costa Rica es importante desde el punto de vista social y económico. Por un lado, es importante identificar las principales causas de muerte de tipo externo y diseñar políticas para reducirlas, por ejemplo, políticas de prevención y salud ocupacional. Los adolescentes y los jóvenes adultos se están uniendo a los sectores productivos de la economía y formando parte del bono demofigura, por lo que el estudio de esa población cobra singular importancia". Bajo ciertas condiciones, Costa Rica puede experimentar cambios positivos de tipo económico debido al cambio en la pirámide poblacional(20).

\section{Materiales y métodos}

Estudio descriptivo, estadístico, demofigura, epidemiológico y comparativo de corte transversal (cross-sectional study), que evalúa la mortalidad entre jóvenes inmigrantes nicaragüenses y la compara con la mortalidad de los costarricenses en Costa Rica. Para la operacionalización de la mortalidad, se siguieron las siguientes definiciones: 
Los numeradores del estudio fueron las defunciones de los jóvenes inmigrantes nicaragüenses y de los costarricenses en un período de 18 años, entre el primero de enero del 2000 y el 31 de diciembre del 2017. La base de datos de las defunciones para los años 2000-2017 fue utilizada como fuente de los datos, la cual está disponible en el Instituto Nacional de Estadística y Censos(3,4). Debido al que los jóvenes tienen los riesgos más altos en muertes debido a causas externas, el análisis del estudio se enfocó en las muertes entre jóvenes entre 15 y 34 años de edad ( $n=23$ 776; costarricenses: $n_{1}=20$ 909; inmigrantes nicaragüenses: $\mathrm{n}_{2}=2$ 867). La base de datos tiene la variable lugar de nacimiento, la cual se utilizó para indicar si la defunción fue de un inmigrante nicaragüense o de un costarricense.

\section{Análisis comparativo}

Para realizar una comparación con el trabajo de Bonilla y Chavarría(1), se utilizó la siguiente clasificación de enfermedades y causas externas utilizando la Clasificación Internacional de Enfermedades, Décima Edición CIE-10(13). Para el caso de las defunciones por enfermedades:

- D1. Enfermedades infecciosas: J00-J42, A17B19, B25-B99.

- D2. Cáncer: C15-C26, C30-C39, C53-C55, C50, C61, C00-14, C40-9, C51-2, C56-60, C62-D48.

- D3. Enfermedades respiratorias crónicas: J43-J99.

- D4. Enfermedades cardiovasculares: I00-I99.

- D5. Diabetes: E10-E14.

- D6. Alcoholismo: F10, K70-K76.

- D7. Otras causas: A00-A09, A15, A16, D50D53, E40-E46, 000-099, P00-P96, Q00-Q99, B20-B24, R54, R95-99 y resto.

- En el caso de las defunciones por causas externas:

- D8. Accidentes vehiculares: V01-V89.

- D9. Suicidios: X60-X84.

- D10. Homicidios: X91-Y34.

- D11. Otros accidentes: W00-X59.

\section{Población}

Los denominadores del estudio utilizados para calcular las tasas de mortalidad estandarizadas por 100 mil habitantes fue la población del X Censo de Población y Vivienda 2011(3,4). Los denominadores fueron definidos de esta forma debido a la falta de estimaciones de población de inmigrantes nicaragüenses para cada año del período 2000-2017. El periodo histórico del estudio entre 2000 y 2017 se definió de esta forma por el hecho de disponer estimaciones de población de inmigrantes nicaragüenses provenientes del Censo de Población 2011. Para controlar el posible efecto de la edad y el sexo, las tasas de mortalidad fueron estandarizadas por la población de los Estados Unidos en el año 2015(14).

\section{Análisis estadístico}

Se realizó análisis de distribución de frecuencias para comparar la importancia relativa de las defunciones entre jóvenes inmigrantes nicaragüenses y su contraparte costarricense (subpoblaciones). Se hizo una comparación con los datos de Bonilla y Chavarría(1) que estudiaron el mismo fenómeno en el periodo 1998-2008 para evaluar los cambios entre esos 10 años. Las tasas de mortalidad por causas de muerte (defunciones por 100 mil habitantes) fueron calculadas para ambas subpoblaciones de la siguiente forma: para cada subpoblación, las defunciones (numeradores) fueron divididas entre la población (denominadores) y multiplicados por 100 mil habitantes.

El riesgo relativo $(R R)$ fue calculado por causa de muerte de los jóvenes inmigrantes nicaragüenses (numerador) con respecto a los costarricenses (denominadores), el RR resulta de dividir cada tasa de mortalidad estandarizada por causa. Los RR mayores que uno, significan que los jóvenes inmigrantes nicaragüenses tienen un riesgo de mortalidad mayor en una causa de muerte específica que su contraparte costarricense. El RR se usa para hacer una comparación entre dos subpoblaciones. Se calculó un intervalo de confianza al 95\% para los RR, bajo el supuesto que las tasas de mortalidad estandarizadas tienen una distribución de Poisson(15).

Los datos fueron procesados con el paquete estadístico STATA 17(16). Finalmente, se compararon las tasas de mortalidad estandarizadas con las de Bonilla y Chavarría(1) para evaluar los cambios entre esos 10 años.

\section{Consideraciones éticas}

En el estudio no se trabajó con seres humanos, sino con las bases de datos públicas y anonimizadas de las estadísticas producidas por el Instituto Nacional de Estadística y Censos, por lo que según el Comité Ético Científico de la Universidad de Costa Rica y la legislación costarricense actual (Ley 9234), la 
presente investigación no requiere un aval ético institucional.

\section{Resultados}

En la Tabla 1 se presentan las defunciones de jóvenes inmigrantes nicaragüenses y costarricenses por causa de muerte. El estudio muestra el $64 \%$ de las defunciones de jóvenes inmigrantes nicaragüenses son por causas externas (homicidios, accidentes vehiculares, otros accidentes y suicidios), versus el $57 \%$ de su contraparte costarricense. Particularmente el $23 \%$ de las defunciones de inmigrantes nicaragüenses se debe a los homicidios, versus el $17 \%$ de los costarricenses, dicha diferencia es significativa al $5 \%(\mathrm{z}=3,75, p<0, .0001)$. En este sentido, los homicidios son la primera causa de muerte entre los inmigrantes nicaragüenses (23\%), igual que en los datos de hace 10 años, sin embargo, entre los costarricenses, este lugar es ocupado por los accidentes vehiculares. Con respecto a los datos de hace 10 años presentados por Bonilla $\mathrm{y}$ Chavarría(1), hay una disminución de 2 puntos porcentuales de las defunciones por causas externas entre los jóvenes inmigrantes nicaragüenses.
En la Tabla 2 y la Figura 1 se presentan las tasas de mortalidad estandarizadas y los riesgos relativos para jóvenes inmigrantes nicaragüenses y costarricenses por causa de muerte. La Tabla 2 muestra también los riesgos relativos por causa de muerte, que resulta de dividir las tasas; un intervalo al 95\% de confianza para esos riesgos relativos y el cambio con respecto a hace 10 años, basados en los riesgos relativos presentados por Bonilla $\mathrm{y}$ Chavarría(1). Con respecto a los riesgos relativos, los valores superiores a uno, indican que los inmigrantes nicaragüenses tienen un riesgo superior por esta causa de muerte con respecto a los costarricenses.

Los inmigrantes tienen RR mayores de mortalidad por causas externas que los costarricenses: otros accidentes 1,85, homicidios 1,72, accidentes vehiculares 1,22 y suicidios 1,14 . Con respecto a hace 10 años, se destaca una disminución de 0,27 y 0,15 del RR de los homicidios y alcoholismo respectivamente. Hay un aumento de 0,12 del RR en cáncer entre los inmigrantes nicaragüenses con respecto a hace 10 años. En la Figura 2 se presentan los RR. En general, los resultados indican que el RR de mortalidad por causas externas entre inmigrantes nicaragüenses y la población costarricense se ha acortado en los últimos 10 años.

Tabla 1. Defunciones de jóvenes inmigrantes nicaragüenses y costarricenses por causa de muerte (Reclasificación de los códigos del CIE-10ª). Costa Rica 2000-2017

\begin{tabular}{|c|c|c|c|c|c|c|}
\hline \multirow{2}{*}{ Causa de muerte } & \multicolumn{3}{|c|}{ Costarricenses } & \multicolumn{3}{|c|}{ Inmigrantes nicaragüenses } \\
\hline & Defunciones & $\%$ & Cambio a $^{\text {a }}$ & Defunciones & $\%$ & Cambio b $^{\text {b }}$ \\
\hline \multicolumn{7}{|l|}{ Enfermedades } \\
\hline Enfermedades infecciosas & 447 & 2,1 & 0,0 & 64 & 2,2 & $-0,2$ \\
\hline Cáncer & 2662 & 12,7 & $-0,6$ & 265 & 9,2 & 0,7 \\
\hline Enfermedades respiratorias crónicas & 282 & 1,3 & $-0,5$ & 30 & 1,0 & $-0,3$ \\
\hline Enfermedades cardiovasculares & 1254 & 6,0 & $-0,2$ & 174 & 6,1 & 0,3 \\
\hline Diabetes & 127 & 0,6 & $-0,1$ & 12 & 0,4 & 0,0 \\
\hline Alcoholismo & 330 & 1,6 & $-0,3$ & 35 & 1,2 & $-0,3$ \\
\hline Otras causas & 3961 & 18,9 & $-1,0$ & 447 & 15,6 & $-1,0$ \\
\hline \multicolumn{7}{|l|}{ Causas externas } \\
\hline Accidentes vehiculares & 4547 & 21,7 & 0,2 & 607 & 21,2 & 0,4 \\
\hline Suicidios & 2153 & 10,3 & $-0,4$ & 256 & 8,9 & $-0,1$ \\
\hline Homicidios & 3534 & 16,9 & 3,7 & 659 & 23,0 & 1,7 \\
\hline Otros accidentes & 1612 & 7,7 & $-1,1$ & 318 & 11,1 & $-1,3$ \\
\hline Total & 20909 & 100 & & 2867 & 100 & \\
\hline
\end{tabular}

a Clasificación Internacional de las Enfermedades, Décima Revisión, CIE-10(13).

b Cambio con respecto al año 2000(1).

Fuente: Elaboración propia con base en los datos de las estadísticas vitales del Instituto Nacional de Estadística y Censos(4) y del Centro Centroamericano de Población(3). 
Tabla 2. Tasas de mortalidad estandarizadas y riesgo relativos para jóvenes inmigrantes nicaragüenses y costarricenses por causa de muerte (Reclasificación de los códigos del CIE-10 a). Costa Rica 2000-2017. (Tasas por 100 mil personas promediadas por sexo)

\begin{tabular}{|c|c|c|c|c|c|c|}
\hline \multirow{2}{*}{ Causa de muerte } & \multicolumn{2}{|c|}{ Inmigrantes } & \multirow{2}{*}{$\begin{array}{c}\text { Riesgo } \\
\text { relativo }\end{array}$} & \multicolumn{2}{|c|}{ Intervalo al 95\% } & \multirow{2}{*}{ Cambio $^{\mathrm{b}}$} \\
\hline & Costarricenses & Nicaragüenses & & $L_{i}$ & $L_{s}$ & \\
\hline \multicolumn{7}{|l|}{ Enfermedades } \\
\hline Enfermedades infecciosas & 1,90 & 2,44 & 1,28 & 1,20 & 1,36 & $-0,06$ \\
\hline Cáncer & 11,35 & 10,22 & 0,90 & 0,88 & 0,93 & 0,12 \\
\hline Enfermedades respiratorias crónicas & 1,20 & 1,11 & 0,93 & 0,85 & 1,01 & 0,02 \\
\hline Enfermedades cardiovasculares & 5,41 & 6,39 & 1,18 & 1,14 & 1,23 & 0,03 \\
\hline Diabetes & 0,57 & 0,45 & 0,80 & 0,70 & 0,92 & 0,07 \\
\hline Alcoholismo & 1,46 & 1,31 & 0,89 & 0,83 & 0,97 & $-0,15$ \\
\hline Otras causas & 16,58 & 16,77 & 1,01 & 0,99 & 1,04 & $-0,01$ \\
\hline \multicolumn{7}{|l|}{ Causas externas } \\
\hline Accidentes vehiculares & 18,84 & 23,03 & 1,22 & 1,20 & 1,25 & 0,05 \\
\hline Suicidios & 8,96 & 10,24 & 1,14 & 1,11 & 1,18 & 0,08 \\
\hline Homicidios & 14,71 & 25,34 & 1,72 & 1,69 & 1,76 & $-0,27$ \\
\hline Otros accidentes & 6,66 & 12,35 & 1,85 & 1,80 & 1,91 & 0,16 \\
\hline
\end{tabular}

a Clasificación Internacional de las Enfermedades, Décima Revisión, CIE-10 ${ }^{(13)}$.

b Cambio con respecto al año $2000^{(1)}$.

Fuente: Elaboración propia con base en los datos de las estadísticas vitales del Instituto Nacional de Estadística y Censos ${ }^{(4)}$ y del Centro Centroamericano de Población ${ }^{(3)}$.

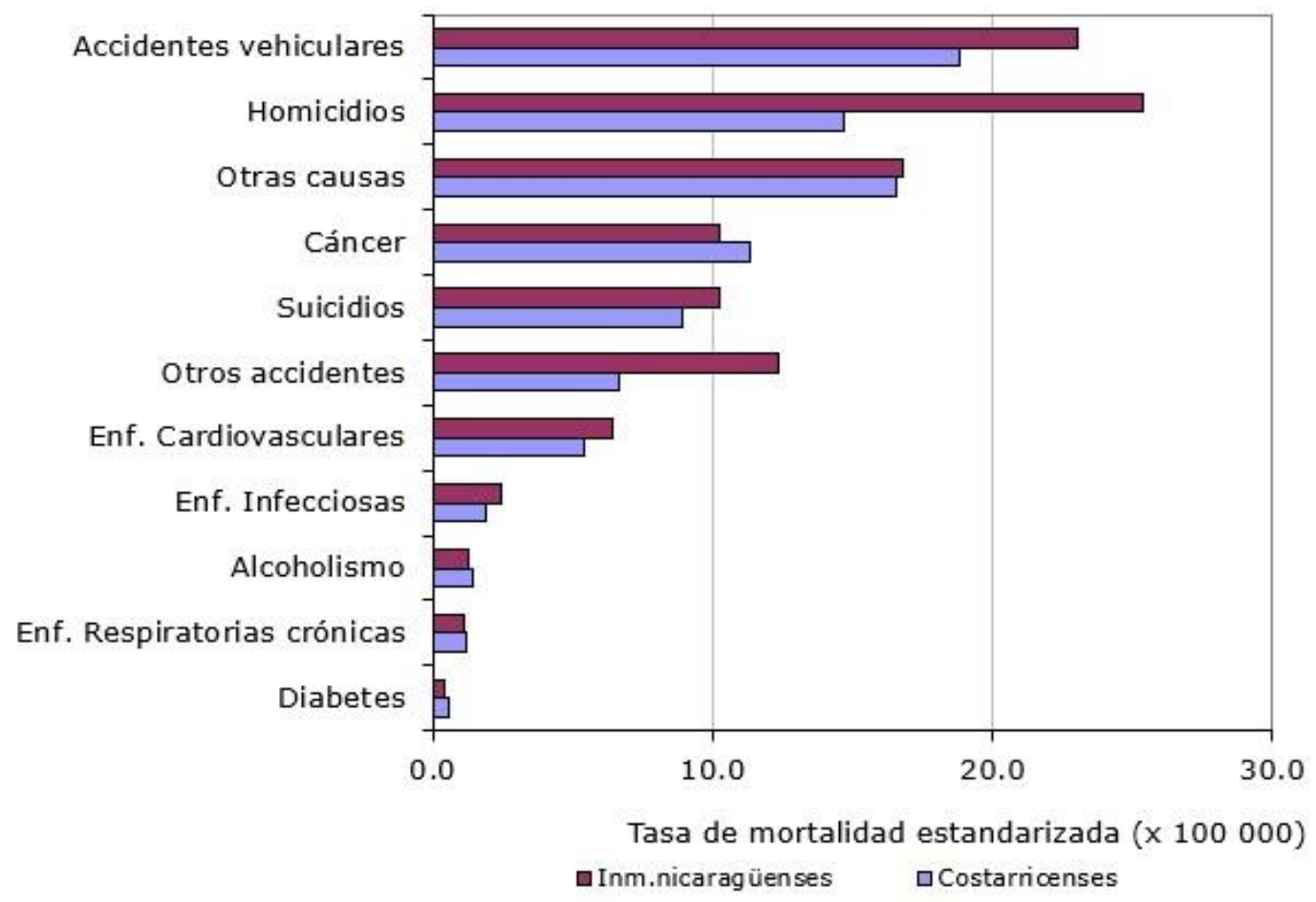

Figura 1. Tasas de mortalidad estandarizadas para jóvenes inmigrantes nicaragüenses y costarricenses por causa de muerte (Reclasificación de los códigos del CIE-10 a). Costa Rica 2000-2017.

Fuente: Elaboración propia con base en los datos de las estadísticas vitales del Instituto Nacional de Estadística y Censos ${ }^{(4)}$ y del Centro Centroamericano de Población ${ }^{(3)}$. 


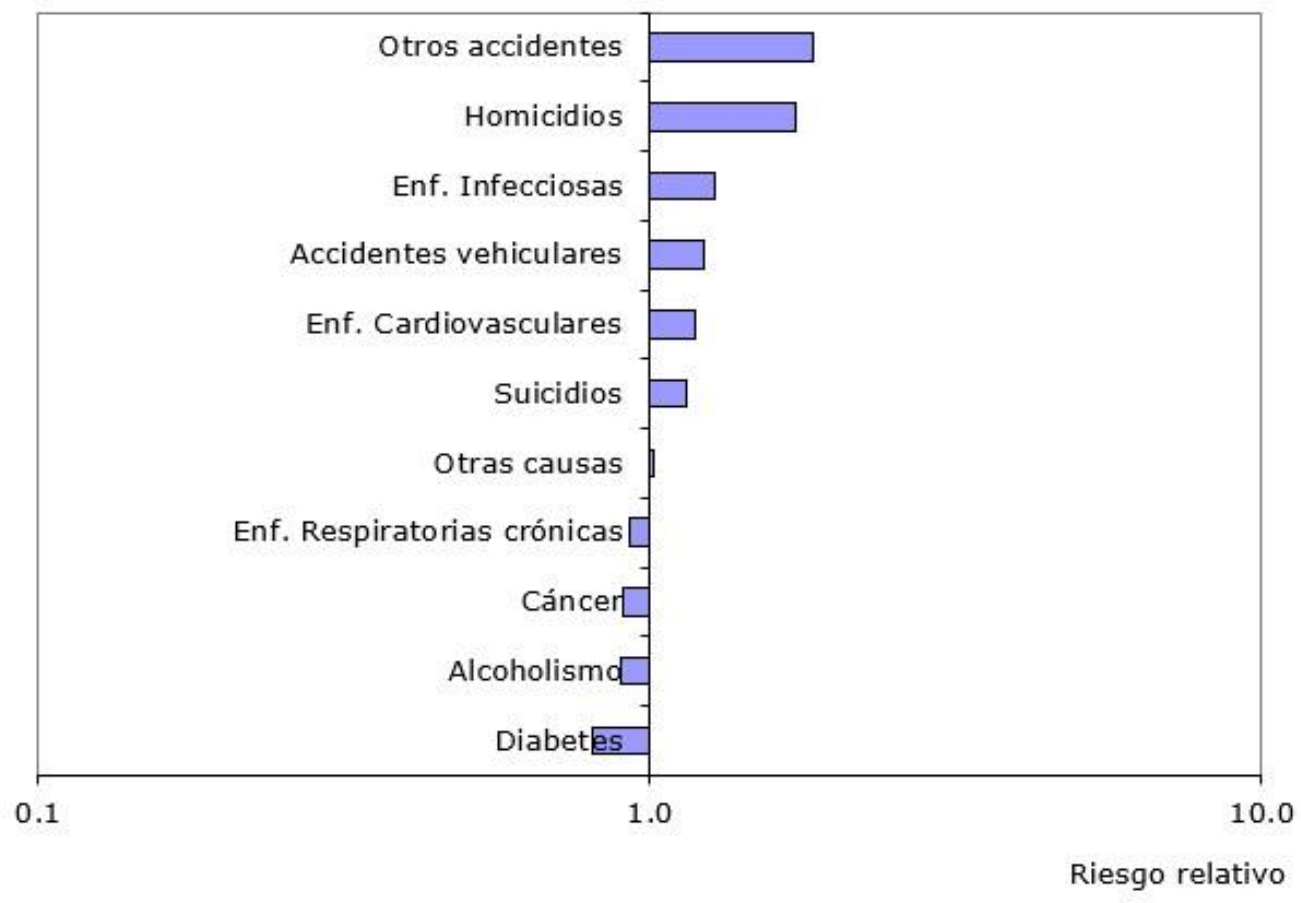

Figura 2. Riesgo Relativos para jóvenes inmigrantes nicaragüenses y Costarricenses por causa de muerte (Reclasificación de los códigos del CIE-10 a). Costa Rica 2000-2017.

(Tasas por 100 mil personas promediadas por sexo).

Fuente: Elaboración propia con base en los datos de las estadísticas vitales del Instituto Nacional de Estadística y Censos ${ }^{(4)}$ y del Centro Centroamericano de Población ${ }^{(3)}$.

\section{Discusión}

Los resultados de este estudio señalan que los jóvenes inmigrantes nicaragüenses tienen mayores riesgos de mortalidad por causas externas que los costarricenses, particularmente en otros accidentes y en homicidios. Esto coincide con lo encontrado en los estudios de Bonilla y Chavarria(1) y Herring et al.,(5) para el caso de Costa Rica; Shor et al.,(7) y Aldridge et al.,(8) en Estados Unidos; Ikram et al.,(9) en Europa, los autores afirman que las muertes por causas violentas y accidentes en la población nicaragüense merecen atención especial.

Entre los inmigrantes se presenta una baja mortalidad y morbilidad en muertes por enfermedades y alta mortalidad en causas externas (particularmente en accidentes y homicidios). Se reafirma el estudio de Acuña et al.,(12) quienes concluyen que el perfil de la mortalidad asociada a enfermedades de la población inmigrante presenta patrones similares al de la población costarricense que evidencia un aumento de muertes por enfermedades crónicas y traumatismos.

Existe una disminución en el RR por homicidios y un aumento por cáncer, con respecto a los datos de hace 10 años. La información de este trabajo sugiere que los patrones de mortalidad de los jóvenes inmigrantes nicaragüenses empiezan comportarse como el de la población costarricense. Es posible que entre los inmigrantes se presente el efecto de la inculturación y la adaptación al sistema de salud y al sistema socioeconómico costarricense, como se observa en otras poblaciones inmigrantes a nivel mundial(21-23), la investigación debe dirigirse a profundizar en este sentido. Una posible hipótesis es que el narcotráfico y las actividades del crimen organizado tiene influencia en los homicidios(17-19).

Lo anterior hace pensar que el fenómeno de la violencia hacia los inmigrantes es un poco más difuso y se presentan nuevos imaginarios de la violencia no analizados. ¿Los jóvenes inmigrantes nicaragüenses tienen menos participación en dichas actividades ilegales que los costarricenses y eso hace 
que se reduzcan los RR por homicidios?, los resultados de este artículo contribuyen a presentar evidencia para un apropiado diseño de políticas públicas en salud y seguridad que podría beneficiar a los jóvenes inmigrantes. Este trabajo presenta la base para futuras investigaciones.

¿Qué tan válidos y confiables son estos resultados? Igual que en el caso del estudio de Bonilla y Chavarría(1), existen dos elementos que podrían reducir la validez de los resultados. En primer lugar, es el subregistro de inmigrantes nicaragüenses, que han vivido menos de seis meses en Costa Rica, debido al hecho que el Censo reportó solamente residentes que han vivido seis meses o más en la vivienda. En segundo lugar, está el efecto del numerador, debido a que el tamaño muestral es pequeño. En este estudio se usa la subpoblación de inmigrantes nicaragüenses en un periodo de tiempo específico para garantizar un número suficiente de casos en el numerador para calcular las tasas.

\section{Conclusiones}

La mortalidad por causas externas predomina entre la población inmigrante nicaragüense en Costa Rica particularmente en otros accidentes, homicidios, accidentes vehiculares y suicidios. Se observa una disminución de 2 puntos porcentuales en la mortalidad por causas externas y la información de este trabajo sugiere que los patrones de mortalidad de los inmigrantes empiezan comportarse como el de la población costarricense. Los resultados de este estudio son la base de futuras investigaciones a fin de diseñar políticas en migración y salud.

Conflicto de intereses: El autor no declara conflictos de interés

\section{Referencias}

1. Bonilla RE, Chavarría JB. Mortality among young Nicaraguan immigrants to Costa Rica: Deaths from disease versus injury. Población y Salud en Mesoam. 2015;13(1). Disponible en: https://revistas.ucr.ac.cr/index.php/psm/article/view/194 97

2. Bonilla-Carrión R. Estado de Salud en Jóvenes Inmigrantes Nicaragüenses en Costa Rica ¿Son tan diferentes? Investigación en Juventudes: Revista del Consejo de la Persona Joven. 2020;7(1):80-101. Disponible en: https://cpj.go.cr/capitulo/estado-de-salud-en-jovenesinmigrantes-nicaraguenses-en-costa-rica-hay-diferenciascon-la-poblacion-local/
3. Centro Centroamericano de Población (CCP). Censo Nacional de Población y Vivienda, Costa Rica. [Internet] [citado el 15 de Junio 2019]. Disponible en: http://censos.ccp.ucr.ac.cr

4. Instituto Nacional de Estadística y Censos (INEC). Estadísticas Vitales de Mortalidad. [Internet] [citado el 15 de Junio 2019]. Disponible en: http://www.inec.go.cr

5. Herring AA, Bonilla RE, Borland RM, Hill KH. Patrones diferenciales de mortalidad entre inmigrantes nicaragüenses y residentes nativos de Costa Rica. Población y Salud en Mesoam. 2008;6(1). Disponible en: https://revistas.ucr.ac.cr/index.php/psm/article/view/453 4

6. Solís L, Chaves N. Experiencias laborales de Jóvenes Migrantes en el sector piñero de la zona norte de Costa Rica. En: Repensar las fronteras, la integración regional y el territorio. Editado por Willy Soto Acosta. 1a. Ed. Heredia, Costa Rica: CLACSO, IDESPO, Universidad Nacional de Costa Rica. 2017. 352 p. ISBN: 978-9968-558-46-4. Disponible en: https://bit.ly/30iPl99

7. Shor E, Roelfs D, Vang ZM. The "Hispanic mortality paradox" revisited: Meta-analysis and meta-regression of life-course differentials in Latin American and Caribbean immigrants' mortality. Social Science \& Medicine. 2017;186:20-33. Disponible en: https://doi.org/10.1016/j.socscimed.2017.05.049

8. Aldridge RW, Nellums LB, Bartlett S, Barr AL, Patel P, Burns $\mathrm{R}$, et al. Global patterns of mortality in international migrants: a systematic review and meta-analysis. The Lancet. 2018;392(10164):2553-66. Disponible en: https://doi.org/10.1016/S0140-6736(18)32781-8

9. Ikram UZ, Malmusi D, Juel K, Rey G, Kunst AE. Association between Integration Policies and Immigrants' Mortality: An Explorative Study across Three European Countries. PLoS ONE. 2015;10(6):e0129916. DOI: 10.1371/journal.pone.0129916

10. Yáñez del V Á. Tuberculosis en inmigrantes: Situación ChilePerú. Revista Chilena de Enfermedades Respiratorias. 2010;26(3):161-4. Disponible en: http://dx.doi.org/10.4067/S0717-73482010000300008

11. Herring A, Bonilla R. Inmigrantes Nicaragüenses en Costa Rica: Estado y Utilización de Servicios de Salud. Población y Salud en Mesoam. 2009;7(1). DOI: 10.15517/psm.v7i1.1094

12. Acuña GE, Morales A, Gómez X, Montiel H. Desarrollo y salud en Costa Rica: Elementos para su análisis. 2003; Organización Panamericana de la Salud. San José, Costa Rica: OPS, 35 p. Disponible en: https://bit.ly/3rxr6jL

13. World Health Organization (WHO). International Statistical Classification of Diseases, 10th Revision (ICD-10). [Internet]. 2019 [citado el 15 de Junio 2019]. Disponible en: https://icd.who.int/browse10/2019/en

14. U.S. Census Bureau (USCB), Population Division. Annual Estimates of the Resident Population for Selected Age Groups by Sex for the United States, States, Counties and Puerto Rico Commonwealth and Municipios: April 1, 2010 to July 1, 2015. [Internet] 2016 [citado el 15 de Agosto 2019]. Disponible en: https://www.census.gov/

15. Díaz-Quijano FA. Regresiones aplicadas al estudio de eventos discretos en epidemiología. Revista Salud UIS. 2016;48(1):9-15. Disponible en: https://doi.org/10.18273/revsal.v48n1-2016001

16. StataCorp. Stata Statistical Software: Release 15. 2017; College Station, TX: StataCorp LLC. Disponible en: https://www.stata.com/ 
17. Sánchez-Hernández L. Patrones Territoriales y Factores Sociodemofiguras asociados a los Homicidios y el Narcotráfico en Costa Rica. Informe final. Estado de la Nación, San José, Costa Rica; 2018. Disponible en: http://hdl.handle.net/20.500.12337/2973

18. Comisión Técnica Interinstitucional sobre Estadísticas de Convivencia y Seguridad Ciudadana (COMESCO). Análisis de los Homicidios Dolosos Vinculados a la Delincuencia Organizada durante el periodo 2010-2016. Informe final. San José, Costa Rica; 2017. Disponible en: https://www.latinamerica.undp.org/content/rblac/es/hom e/library/democratic_governance/analisis-de-loshomicidios-dolorosos-vinculados-a-la-delincuenci.html

19. Vargas-Gutiérrez U. Chapter III. Homicides in Mexico. Jalisco on the Violence Map. En: Moloeznik MB, Rodríguez A, compiladores. Security and Justice in Jalisco: Scenario and Proposals. Consejo Estatal de Ciencia y Tecnología de Jalisco (COECYTJAL). México; 2016. Disponible en: https://www.worldcat.org/title/security-and-justice-injalisco-scenarios-and-proposals/oclc/1022119759
20. Jiménez-Fontana P. Retos para materializar el dividendo de género perfiles de uso de tiempo en Costa Rica. Población y Salud en Mesoamérica. 2016;13(2). Disponible en: http://dx.doi.org/10.15517/psm.v13i2.21748

21. Huang KY, Calzada E, Cheng S, Barajas-Gonzales RG, Miller-Brotmanet L. Cultural Adaptation, Parenting and Child Mental Health Among English Speaking Asian American Immigrant Families. Child Psychiatry Hum Dev. 2017;48(4):572-83. Disponible https://doi.org/10.1007/s10578-016-0683-y

22. Kwak K. Age and Gender Variations in Healthy Immigrant Effect: a Population Study of Immigrant Well-Being in Canada. Int. Migration \& Integration. 2018;19(2):413-37. Disponible en: https://doi.org/10.1007/s12134-018-05464

23. Kennedy S, Kidd MP, McDonald JT, Biddle N. The healthy immigrant effect: patterns and evidence from four countries. Journal of International Migration and Integration. 2015;16(2):317-32. Disponible en: https://doi.org/10.1007/s12134-014-0340-x 